\title{
Research Article \\ Computing Exact Solutions to a Generalized Lax-Sawada-Kotera-Ito Seventh-Order KdV Equation
}

\author{
Alvaro H. Salas, ${ }^{1,2}$ Cesar A. Gómez $S^{3}$ \\ and Bernardo Acevedo Frias ${ }^{4}$ \\ ${ }^{1}$ Universidad de Caldas, Calle 65 No. 26-10, P.O. Box: Apartado Aéreo 275, Manizales, Caldas, Colombia \\ ${ }^{2}$ Department of Mathematics, Universidad Nacional de Colombia, Carrera 27 No. 64-60, P.O. Box: \\ Apartado Aéreo 275, Manizales, Colombia \\ ${ }^{3}$ Department of Mathematics, Universidad Nacional de Colombia, Calle 45, Carrera 30. P.O. Box: \\ Apartado Aéreo 52465, Bogota, Colombia \\ ${ }^{4}$ Department of Mathematics, Universidad Nacional de Colombia, P.O. Box: Apartado Aéreo 127, \\ Manizales, Colombia
}

Correspondence should be addressed to Alvaro H. Salas, asalash2002@yahoo.com

Received 24 December 2009; Revised 5 May 2010; Accepted 4 August 2010

Academic Editor: Katica R. (Stevanovic) Hedrih

Copyright (C) 2010 Alvaro H. Salas et al. This is an open access article distributed under the Creative Commons Attribution License, which permits unrestricted use, distribution, and reproduction in any medium, provided the original work is properly cited.

The Cole-Hopf transform is used to construct exact solutions to a generalization of both the seventh-order Lax KdV equation (Lax KdV7) and the seventh-order Sawada-Kotera-Ito KdV equation (Sawada-Kotera-Ito KdV7).

\section{Introduction}

Many direct and computational methods have been used to handle nonlinear partial differential equations (NLPDE's). Some methods used in a satisfactory way to obtain exact solutions to NLPDE's are inverse scattering method [1], Hirota bilinear method [2, 3], Backlund transformations [4], Painlevé analysis [5], Lie groups [6], the tanh method [7], the generalized tanh method $[8,9]$, the extended tanh method [10-12], the improved tanh-coth method [13, 14], the Exp-function method [15-17], the projective Riccati equation method [18], the generalized projective Riccati equations method [19-24], the extended hyperbolic function method [25], variational iteration method [26, 27], He's polynomials [28], homotopy perturbation method [29], and many other methods [30]. However, there is not a unified method that could be used to handle all NLPDE's; in this sense, the implementation of new 
methods or variants of the some well-known methods is relevant. The principal objective of this paper consists in obtaining exact traveling wave solutions which include periodic and soliton solutions to a particular case of the general seventh-order KdV (KdV7), which is a generalization of the seventh-order Sawada-Kotera-Ito (SKI-KdV7) equation, by using a variant of the exp-function method. The general seventh-order KdV (KdV7) equation [31] reads

$$
u_{t}+a u^{3} u_{x}+b u_{x}^{3}+c u u_{x} u_{x x}+d u^{2} u_{x x x}+e u_{2 x} u_{3 x}+f u_{x} u_{4 x}+g u u_{5 x}+u_{7 x}=0 .
$$

The (KdV7) was introduced initially by Pomeau et al. [32] for discussing the structural stability of $\mathrm{KdV}$ equation under a singular perturbation. Some particular cases of (1.1) are

(i) seventh-order Lax equation $[1,6](a=140, b=70, c=280, d=70, e=70, f=42$, $g=14)$ :

$$
u_{t}+140 u^{3} u_{x}+70 u_{x}^{3}+280 u u_{x} u_{x x}+70 u^{2} u_{x x x}+70 u_{2 x} u_{3 x}+42 u_{x} u_{4 x}+14 u u_{5 x}+u_{7 x}=0
$$

(ii) seventh-order Sawada-Kotera-Ito equation $[1,8-10](a=252, b=63, c=378$, $d=126, e=63, f=42, g=21)$ :

$$
u_{t}+252 u^{3} u_{x}+63 u_{x}^{3}+378 u u_{x} u_{x x}+126 u^{2} u_{x x x}+63 u_{2 x} u_{3 x}+42 u_{x} u_{4 x}+21 u u_{5 x}+u_{7 x}=0
$$

(iii) seventh-order Kaup-Kupershmidt equation $[1,7](a=2016, b=630, c=2268$, $d=504, e=252, f=147, g=42)$ :

$$
u_{t}+2016 u^{3} u_{x}+630 u_{x}^{3}+2268 u u_{x} u_{x x}+504 u^{2} u_{x x x}+252 u_{2 x} u_{3 x}+147 u_{x} u_{4 x}+42 u u_{5 x}+u_{7 x}=0
$$

\section{Generalization of the Lax KdV7 and the Sawada-Kotera-Ito KdV7}

Observe that (1.2) and (1.3) satisfy the relation

$$
a=\frac{d}{63}(e+f+g)
$$

For this reason we will study equation

$$
u_{t}+\frac{d}{63}(e+f+g) u^{3} u_{x}+b u_{x}^{3}+c u u_{x} u_{x x}+d u^{2} u_{x x x}+e u_{2 x} u_{3 x}+f u_{x} u_{4 x}+g u u_{5 x}+u_{7 x}=0
$$

We seek solutions to (2.2) in the Cole-Hopf form

$$
u(t, x)=A \partial_{x} \tanh (\xi)
$$


where $A$ is some constant to be determined later and

$$
\xi=\xi(t, x)=\mu(x+\lambda t+\delta), \quad \mu, \delta, \lambda=\text { const. }
$$

Substituting (2.3) into (2.2), we obtain a polynomial equation in the variable $\zeta=\exp (\xi)$. Equating the coefficients of the different powers of $\zeta$ to zero, we obtain following algebraic system:

$$
\begin{gathered}
\lambda+64 \mu^{6}=0 \\
64 \mu^{5}(A(e+f+g)-247 \mu)+5 \lambda=0, \\
64 \mu^{4}\left(A^{2}(b+c+d)-3 A \mu(5 e+9 f+19 g)+4293 \mu^{2}\right)+9 \lambda=0 \\
64 \mu^{3}\left(A^{3} d(e+f+g)-63 A^{2} \mu(3 b+5 c+11 d)+126 A \mu^{2}(28 e+46 f+151 g)-983997 \mu^{3}\right)+315 \lambda=0 .
\end{gathered}
$$

Eliminating $A, \lambda$, and $\mu$ from system (2.5) gives

$$
\begin{gathered}
b=d+\frac{1}{126}(e+f+g)(e-5 f+10 g), \\
c=\frac{5}{21} g(e+f+g)-2 d .
\end{gathered}
$$

It is easy to verify that (1.2) and (1.3) are particular cases of general KdV7 equation (1.1) subject to (2.1) and (2.6). This motivates us to define the generalized Lax-Sawada-Kotera-Ito seventh-order equation (LSKI KdV7) as follows:

$$
\begin{aligned}
u_{t} & +\frac{1}{63} d(e+f+g) u^{3} u_{x}+\left(d+\frac{1}{126}(e+f+g)(e-5 f+10 g)\right) u_{x}^{3} \\
& +\left(\frac{5}{21} g(e+f+g)-2 d\right) u u_{x} u_{x x}+d u^{2} u_{x x x}+e u_{2 x} u_{3 x}+f u_{x} u_{4 x}+g u u_{5 x}+u_{7 x}=0 .
\end{aligned}
$$

\section{Solutions to Generalized LSKI KdV7}

In order to look for solutions to (2.7), we will use the exp ansatz

$$
u(\xi)=p+\frac{q}{1+r \exp (-\xi)+s \exp (\xi)},
$$

where $p, q, r$, and $s$ are some constants. Substituting (3.1) into (2.7) gives an algebraic system. Solving it, we obtain

$$
\lambda=-\frac{1}{63} d(e+f+g) p^{3}-\mu^{2}\left(d p^{2}+g p \mu^{2}+\mu^{4}\right), \quad q=\frac{126 \mu^{2}}{e+f+g}, \quad s=\frac{1}{4 r}, \quad r=r, \quad \mu=\mu .
$$


From (2.4), (3.1), and (3.2), we obtain following solution to (2.7) subject:

$$
\begin{gathered}
u(x, t)=p+\frac{126 \mu^{2}}{(e+f+g)(1+r \exp (\xi)+(1 / 4 r) \exp (-\xi))}, \\
\xi=\mu(x+\lambda t+\delta), \\
\lambda=-\frac{1}{63} d(e+f+g) p^{3}-\mu^{2}\left(d p^{2}+g p \mu^{2}+\mu^{4}\right) .
\end{gathered}
$$

In particular, if $r=1 / 2$, equation (3.3) gives

$$
\begin{gathered}
u(x, t)=p+\frac{63 \mu^{2}}{e+f+g} \operatorname{sech}^{2}\left(\frac{\mu}{2}(x+\lambda t+\delta)\right), \\
\lambda=-\frac{1}{63} d(e+f+g) p^{3}-\left(d p^{2}+g p \mu^{2}+\mu^{4}\right) \mu^{2} .
\end{gathered}
$$

Replacing $\mu$ with $\mu \sqrt{-1}$ gives the following periodic solutions:

$$
\begin{gathered}
u(x, t)=p-\frac{63 \mu^{2}}{e+f+g} \sec ^{2}\left(\frac{\mu}{2}(x+\lambda t+\delta)\right), \\
\lambda=-\frac{1}{63} d(e+f+g) p^{3}+\left(d p^{2}-g p \mu^{2}+\mu^{4}\right) \mu^{2} .
\end{gathered}
$$

On the other hand, if $r=-1 / 2$, equation (3.3) gives

$$
\begin{gathered}
u(x, t)=p-\frac{63 \mu^{2}}{e+f+g} \operatorname{csch}^{2}\left(\frac{\mu}{2}(x+\lambda t+\delta)\right), \\
\lambda=-\frac{1}{63} d(e+f+g) p^{3}-\left(d p^{2}+g p \mu^{2}+\mu^{4}\right) \mu^{2} .
\end{gathered}
$$

Replacing $\mu$ with $\mu \sqrt{-1}$ gives the following periodic solutions:

$$
\begin{gathered}
u(x, t)=p-\frac{63 \mu^{2}}{e+f+g} \csc ^{2}\left(\frac{\mu}{2}(x+\lambda t+\delta)\right), \\
\lambda=-\frac{1}{63} d(e+f+g) p^{3}+\left(d p^{2}-g p \mu^{2}+\mu^{4}\right) \mu^{2} .
\end{gathered}
$$




\section{Solutions to Sawada-Kotera-Ito KdV7 Equation}

From (3.3)-(3.7) with $d=126, e=63, f=42$, and $g=21$, we obtain the following analytic solutions to equation (1.3):

$$
\begin{array}{ll}
u(x, t)=p+\frac{4 r \mu^{2} \exp (\mu(x+\lambda t+\delta))}{(1+2 r \exp (\mu(x+\lambda t+\delta)))^{2}}, \quad \lambda=-252 p^{3}-126 p^{2} \mu^{2}-21 p \mu^{4}-\mu^{6}, \\
u(x, t)=p+\frac{1}{2} \mu^{2} \operatorname{sech}^{2}\left(\frac{1}{2} \mu(x+\lambda t+\delta)\right), \quad \lambda=-252 p^{3}-126 p^{2} \mu^{2}-21 p \mu^{4}-\mu^{6} \\
u(x, t)=p-\frac{1}{2} \mu^{2} \sec ^{2}\left(\frac{1}{2} \mu(x+\lambda t+\delta)\right), \quad \lambda=-252 p^{3}+126 p^{2} \mu^{2}-21 p \mu^{4}+\mu^{6} \\
u(x, t)=p-\frac{1}{2} \mu^{2} \operatorname{csch}^{2}\left(\frac{1}{2} \mu(x+\lambda t+\delta)\right), \quad \lambda=-252 p^{3}-126 p^{2} \mu^{2}-21 p \mu^{4}-\mu^{6} .
\end{array}
$$

\section{Conclusions}

We exhibited an equation that generalizes both seventh-order Lax equation and seventhorder Sawada-Kotera-Ito equation. At the same time, we obtained exact solutions to these equations with the aid of a Cole-Hopf ansatz. These same ideas are suitable for the seventhorder Kaup-Kupershmidt equation. We think that some of the solutions in this work are new in the open literature. We may apply other methods to find exact solutions to a variety of nonlinear PDE's. See [3, 12-52].

\section{References}

[1] M. J. Ablowitz and P. A. Clarkson, Solitons, Nonlinear Evolution Equations and Inverse Scattering, vol. 149 of London Mathematical Society Lecture Note Series, Cambridge University Press, Cambridge, Uk, 1991.

[2] R. Hirota, Direct Methods in Soliton Theory, Springer, Berlin, Germany, 1980.

[3] A. H. Salas, "Exact solutions to mKdV equation with variable coefficients," Applied Mathematics and Computation, vol. 216, no. 10, pp. 2792-2798, 2010.

[4] A. Karasu and S. Yu. Sakovich, "Bäcklund transformation and special solutions for the DrinfeldSokolov-Satsuma-Hirota system of coupled equations," Journal of Physics. A, vol. 34, no. 36, pp. 73557358, 2001.

[5] J. Weiss, M. Tabor, and G. Carnevale, “The Painlevé property for partial differential equations," Journal of Mathematical Physics, vol. 24, no. 3, pp. 522-526, 1983.

[6] P. J. Olver, Applications of Lie Groups to Differential Equations, vol. 107 of Graduate Texts in Mathematics, Springer, New York, NY, USA, 2nd edition, 1993.

[7] E. Fan and Y. C. Hon, "Generalized tanh method extended to special types of nonlinear equations," Zeitschrift für Naturforschung, vol. 57, no. 8, pp. 692-700, 2002.

[8] C. A. Gómez, "Exact solutions for a new fifth-order integrable system," Revista Colombiana de Matematicas, vol. 40, pp. 119-125, 2006.

[9] C. A. Gómez and A. H. Salas, "Exact solutions for a reaction diffusion equation by using the generalized tanh method," Scientia et Technica, vol. 13, no. 35, pp. 409-410, 2007.

[10] A.-M. Wazwaz, "The extended tanh method for new solitons solutions for many forms of the fifthorder KdV equations," Applied Mathematics and Computation, vol. 184, no. 2, pp. 1002-1014, 2007.

[11] C. A. Gómez, "Special forms of the fifth-order KdV equation with new periodic and soliton solutions," Applied Mathematics and Computation, vol. 189, no. 2, pp. 1066-1077, 2007. 
[12] C. A. Gómez, "New exact solutions for a generalization of the Korteweg-de Vries equation (KdV6)," Applied Mathematics and Computation, vol. 216, no. 1, pp. 357-360, 2010.

[13] C. A. Gómez and A. H. Salas, "The generalized tanh-coth method to special types of the fifth-order KdV equation," Applied Mathematics and Computation, vol. 203, no. 2, pp. 873-880, 2008.

[14] A. H. Salas and C. A. Gomez, "Computing exact solutions for some fifth KdV equations with forcing term," Applied Mathematics and Computation, vol. 204, no. 1, pp. 257-260, 2008.

[15] S. Zhang, "Exp-function method exactly solving the KdV equation with forcing term," Applied Mathematics and Computation, vol. 197, no. 1, pp. 128-134, 2008.

[16] J.-H. He and L.-N. Zhang, "Generalized solitary solution and compacton-like solution of the JaulentMiodek equations using the Exp-function method," Physics Letters. A, vol. 372, no. 7, pp. 1044-1047, 2008.

[17] A. H. Salas, "Exact solutions for the general fifth KdV equation by the exp function method," Applied Mathematics and Computation, vol. 205, no. 1, pp. 291-297, 2008.

[18] R. Conte and M. Musette, "Link between solitary waves and projective Riccati equations," Journal of Physics. A, vol. 25, no. 21, pp. 5609-5623, 1992.

[19] Z. Yan, "The Riccati equation with variable coefficients expansion algorithm to find more exact solutions of nonlinear differential equations," MMRC, AMSS, Academis Sinica, vol. 22, no. 1, pp. 275284, 2003.

[20] E. Yomba, "The general projective Riccati equations method and exact solutions for a class of nonlinear partial differential equations," Chinese Journal of Physics, vol. 43, no. 6, pp. 991-1003, 2005.

[21] C. A. Gómez, "New traveling waves solutions to generalized Kaup-Kupershmidt and Ito equations," Applied Mathematics and Computation, vol. 216, no. 1, pp. 241-250, 2010.

[22] C. A. Gómez and A. H. Salas, "Exact solutions for the generalized shallow water wave equation by the general projective Riccati equations method," Boletín de Matemáticas. Nueva Serie, vol. 13, no. 1, pp. 50-56, 2006.

[23] C. A. Gomez and A. H. Salas, "New exact solutions for the combined sinh-cosh-Gordon equation," Lecturas Matemáticas, vol. 27, pp. 87-93, 2006.

[24] C. A. Gómez, "New exact solutions of the Mikhailov-Novikov-Wang System," International Journal of Computer, Mathematical Sciences and Applications, vol. 1, pp. 137-143, 2007.

[25] Y. Shang, Y. Huang, and W. Yuan, "New exact traveling wave solutions for the Klein-GordonZakharov equations," Computers \& Mathematics with Applications, vol. 56, no. 5, pp. 1441-1450, 2008.

[26] C. A. Gómez and A. H. Salas, "The variational iteration method combined with improved generalized tanh-coth method applied to Sawada-Kotera equation," Applied Mathematics and Computation. In Press.

[27] M. A. Noor and S.T. Mohyud-Din, "Variational iteration method for solving higher-order nonlinear boundary value problems using He's polynomials," International Journal of Nonlinear Sciences and Numerical Simulation, vol. 9, no. 2, pp. 141-156, 2008.

[28] S. T. Mohyud-Din, M. A. Noor, and K. I. Noor, "Travelling wave solutions of seventh-order generalized KdV equations using He's polynomials," International Journal of Nonlinear Sciences and Numerical Simulation, vol. 10, no. 2, pp. 223-229, 2009.

[29] S. T. Mohyud-Din and M. A. Noor, "Homotopy perturbation method for solving partial differential equations," Zeitschrift für Naturforschung, vol. 64, no. 3-4, pp. 157-170, 2009.

[30] S. T. Mohyud-Din, M. A. Noor, and K. I. Noor, "Some relatively new techniques for nonlinear problems," Mathematical Problems in Engineering, vol. 2009, Article ID 234849, p. 25, 2009.

[31] U. Göktaş and W. Hereman, "Symbolic computation of conserved densities for systems of nonlinear evolution equations," Journal of Symbolic Computation, vol. 24, no. 5, pp. 591-622, 1997.

[32] Y. Pomeau, A. Ramani, and B. Grammaticos, "Structural stability of the Korteweg-de Vries solitons under a singular perturbation," Physica D, vol. 31, no. 1, pp. 127-134, 1988.

[33] P. D. Lax, "Integrals of nonlinear equations of evolution and solitary waves," Communications on Pure and Applied Mathematics, vol. 21, pp. 467-490, 1968.

[34] K. Sawada and T. Kotera, "A method for finding $N$-soliton solutions of the K.d.V. equation and K.d.V.-like equation," Progress of Theoretical Physics, vol. 51, pp. 1355-1367, 1974.

[35] M. Ito, "An extension of nonlinear evolution equations of the K-dV (mK-dV) type to higher orders," Journal of the Physical Society of Japan, vol. 49, no. 2, pp. 771-778, 1980.

[36] P. J. Caudrey, R. K. Dodd, and J. D. Gibbon, "A new hierarchy of Korteweg-de Vries equations," Proceedings of the Royal Society. London. Series A, vol. 351, no. 1666, pp. 407-422, 1976.

[37] D. J. Kaup, "On the inverse scattering problem for cubic eigenvalue problems of the class $\psi_{x x x}+6 Q \psi_{x}+$ $6 R \psi=\lambda \psi, "$ Studies in Applied Mathematics, vol. 62, no. 3, pp. 189-216, 1980. 
[38] A. H. Salas, "Some solutions for a type of generalized Sawada-Kotera equation," Applied Mathematics and Computation, vol. 196, no. 2, pp. 812-817, 2008.

[39] A. H. Salas, "Symbolic computation of solutions for a forced Burgers equation," Applied Mathematics and Computation, vol. 216, no. 1, pp. 18-26, 2010.

[40] A. H. Salas and C. A. Gómez, "Exact solutions for a third-order KdV equation with variable coefficients and forcing term," Mathematical Problems in Engineering, vol. 2009, Article ID 737928, 13 pages, 2009.

[41] A. H. Salas and C. A. Gómez, "Application of the cole-hopf transformation for finding exact solutions to several forms of the seventh-order kdv equation," Mathematical Problems in Engineering, vol. 2010, Article ID 194329, 14 pages, 2010.

[42] A. H. Salas and C. A. Gómez, "A practical approach to solve coupled systems of nonlinear PDE's," Journal of Mathematical Sciences. Advances and Applications, vol. 3, no. 1, pp. 101-107, 2009.

[43] A. H. Salas, "New solutions to Korteweg-de Vries (KdV) equation by the Riccati equation expansion method," International Journal of Applied Mathematics, vol. 22, no. 7, pp. 1169-1177, 2009.

[44] A. H. Salas, "Exact solutions to coupled sine-Gordon equation," Nonlinear Analysis: Real World Applications, vol. 11, no. 5, pp. 3930-3935, 2010.

[45] A. H. Salas, "Symbolic computation of exact solutions to KdV equation," Canadian Applied Mathematics Quarterly, vol. 16, no. 4, 2008.

[46] A. H. Salas, C. A. Gómez, and L. L. Palomá, "Travelling wave solutions to reduced ostrovsky equation," IJAM, vol. 23, pp. 249-256, 2010.

[47] C. A. Gómez, A. H. Salas, and F. B. Acevedo, "Exact solutions to KdV6 equation by the projective Riccati equation method," Mathematical Problems in Engineering. In press.

[48] C. A. Gómez and A. H. Salas, "A generalized Riccati method to solve NLPDE's," Far East Journal of Applied Mathematics, vol. 40, no. 2, 89 pages, 2010.

[49] C. A. Gómez and A. H. Salas, "Special symmetries to standard Riccati equations and applications," Applied Mathematics and Computation, vol. 216, no. 10, pp. 3089-3096, 2010.

[50] J. E. Castillo, A. H. Salas, and C. A. Gómez, "Exact solutions for a nonlinear model," Applied Mathematics and Computation. In press.

[51] A. H. Salas, "Computing exact solutions to a generalized Lax seventh-order forced KdV equation (KdV7)," Applied Mathematics and Computation, vol. 216, no. 8, pp. 2333-2338, 2010.

[52] A. H. Salas, C. A. Gomez S., and J. E. Castillo, "Symbolic computation of solutions for the general fifth-order $\mathrm{KdV}$ equation by using the projective Riccati equation method," International Journal of Nonlinear Science, vol. 9, no. 4, 2010. 


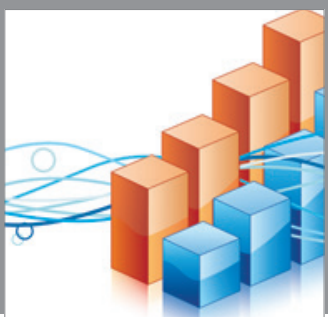

Advances in

Operations Research

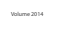

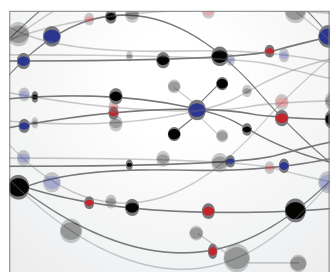

\section{The Scientific} World Journal
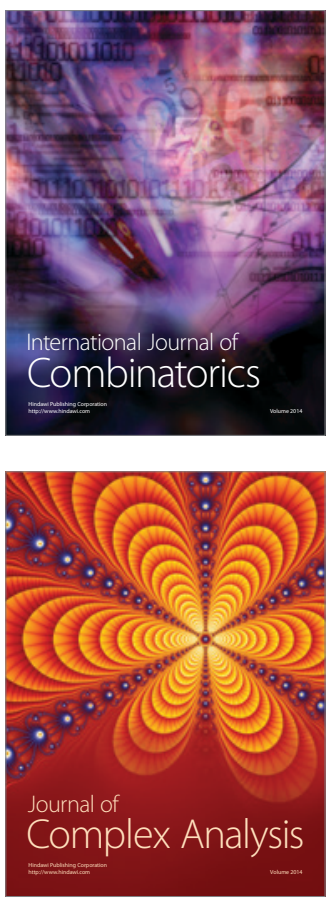

International Journal of

Mathematics and

Mathematical

Sciences
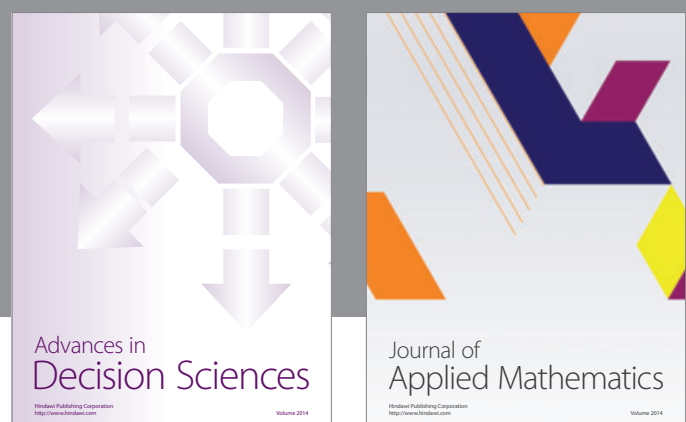

Journal of

Applied Mathematics
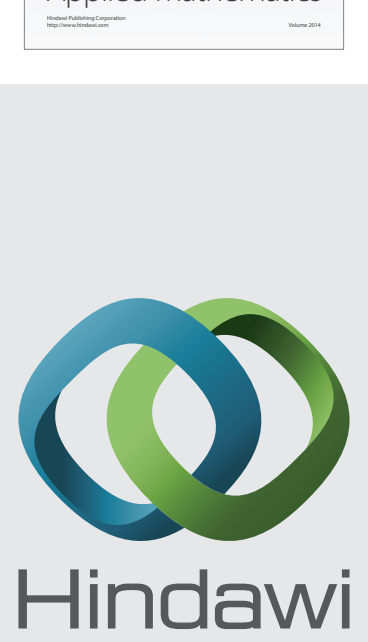

Submit your manuscripts at http://www.hindawi.com
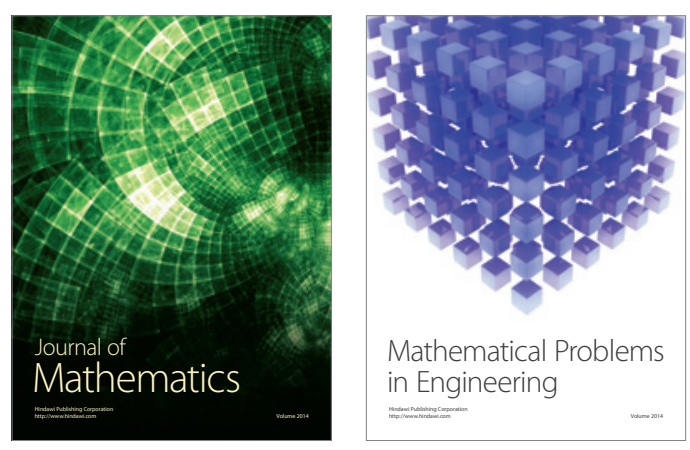

Mathematical Problems in Engineering
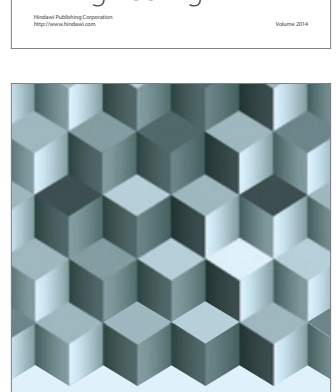

Journal of

Function Spaces
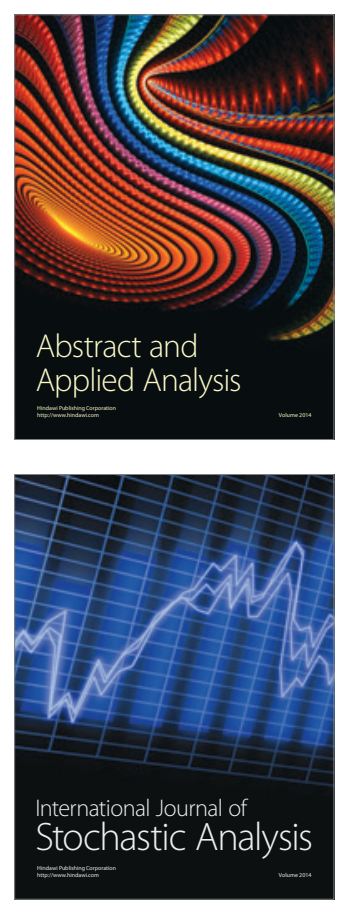

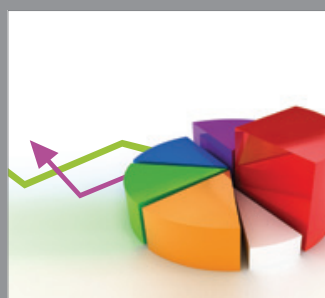

ournal of

Probability and Statistics

Promensencen
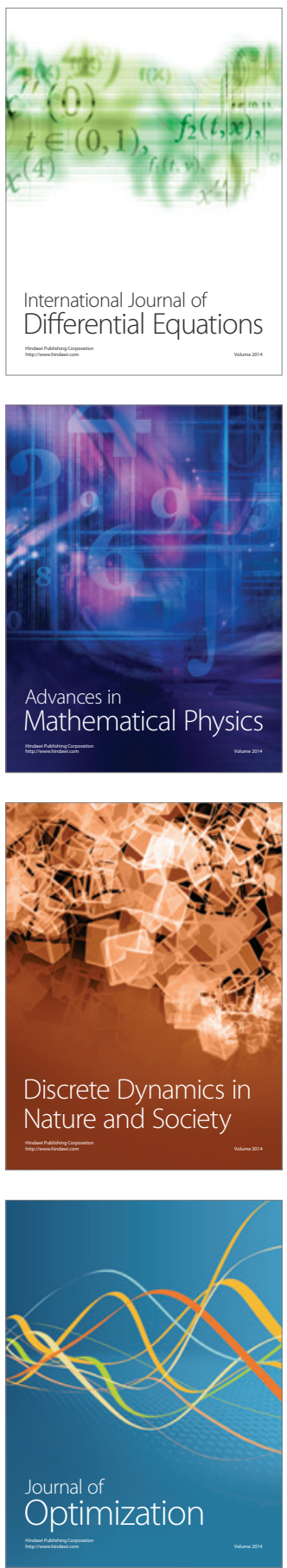